\title{
Adjuvant and definitive radiation therapy for primary carcinoma of the vagina using brachytherapy and external beam radiation therapy
}

\author{
Christopher S. Platta, MD, Bethany Anderson, MD, Heather Geye, MS, Rupak Das, PhD, Margaret Straub, PA-C, \\ Kristin Bradley, MD \\ Department of Human Oncology, University of Wisconsin, Paul P. Carbone Comprehensive Cancer Center, Madison, Wisconsin, USA
}

\begin{abstract}
Purpose: To report the outcomes of patients receiving vaginal brachytherapy and/or external beam radiation therapy (EBRT) for primary vaginal cancer.

Material and methods: Between 1983 and 2009, 63 patients received brachytherapy and/or EBRT for primary tumors of the vagina at a single tertiary center. Patient data was collected via chart review. The Kaplan-Meier method was used to calculate actuarial pelvic local control (LC), disease-free survival (DFS), overall survival (OS), and severe late toxicity rates. Acute and late toxicities were scored according to the Common Terminology Criteria for Adverse Events version 3 (CTCAE v3.0).

Results: Median follow up was 44.2 months. Patients with early stage disease (stages I and II) had significantly improved 5-year OS when compared to patients with locally advanced disease (stages III and IVA) (73.3 vs. 34.4\%, $p=0.032$ ). Patients with greater than $1 / 3$ vaginal involvement had significantly worse prognosis than patients with tumors involving $1 / 3$ or less of the vagina, with the later having superior DFS ( 84.0 vs. $52.4 \%, p=0.007)$ and LC ( 86.9 vs. $60.4 \%, p=0.018)$ at 5-years. Age, histology, and brachytherapy technique did not impact treatment outcomes. The 5-year actuarial grade 3 or higher toxicity rate was $23.1 \%$ (95\% CI: $10.6-35.6 \%)$. Concurrent chemotherapy had no impact on outcomes or toxicity in this analysis.

Conclusions: Success of treatment for vaginal cancer depends primarily on disease stage, but other contributing factors such as extent of vaginal involvement and tumor location significantly impact outcomes. Treatment of vaginal cancer with primary radiotherapy yields acceptable results with reasonable toxicity rates. Management of this rare malignancy requires a multidisciplinary approach to appropriately optimize therapy.

Key words: brachytherapy, radiation therapy, vaginal cancer.

\section{Purpose}

Primary vaginal cancer accounts for less than $2 \%$ of gynecologic malignancies [1,2] with an estimated 2300 cases and 780 deaths in the United States in 2010 [3]. Appropriate evaluation includes a thorough multidisciplinary assessment at diagnosis, with close attention to defining the size and location of the primary tumor as well as any associated regional or distant metastatic spread. Given the close proximity of the vagina to critical pelvic structures such as the bladder, urethra, and rectum, curative surgical resection is often a challenge while assuring appropriate organ sparing and acceptable morbidity [4]. Thus, the primary treatment modality is typically radiation therapy (RT), uti- lizing external beam radiation (EBRT) and/or brachytherapy techniques $[1,2,5,6]$. Concurrent chemotherapy is often employed for patients with locally advanced disease [2].

The rarity of vaginal cancer has prohibited the execution of prospective, randomized trials to define the optimal treatment approach. Treatment decisions are guided by retrospective single-institution series from high-volume centers investigating prognostic factors specifically related to tumor characteristics and treatment techniques [1,2,4,5,7-10]. Here, we report our institutional 26-year experience in treating patients with primary vaginal cancer, analyzing the impact of tumor characteristics and treatment techniques on patient outcomes including local control (LC), disease-free survival (DFS), overall survival (OS), and toxicity rates. 
Table 1. FIGO stage description for vaginal cancer with patient distribution by disease stage

\begin{tabular}{clll} 
Stage & Description & $n(\%)$ & $24(38.1)$ \\
\hline I & Tumor confined to the vagina & $24(38.1)$ & $9(14.3)$ \\
\hline II & Tumor invades paravaginal tissue, but not to pelvic wall & $6(9.5)$
\end{tabular}

FIGO - International Federation of Gynecology and Obstetrics

\section{Material and methods}

\section{Patient population and treatment methods}

From 1983 to 2009, a total of 63 patients were treated for primary carcinoma of the vagina at a tertiary medical facility. At diagnosis, all patients were evaluated in a multidisciplinary fashion by a radiation oncologist and a gynecologic oncologist. Clinical examination was performed to determine the extent of primary tumor, including examination under anesthesia, if indicated. The pretreatment staging workup varied over the 26-year period encompassed by this study, with many patient receiving several of the following: CT (computed tomography) scan, MRI (magnetic resonance imaging) scan, chest X-ray, PET (positron emission tomography) scan, cystoscopy, exam under anesthesia, colposcopy, proctoscopy, and routine blood work. Stage was defined per the International Federation of Gynecology (FIGO) staging system (Table 1).

All patients in this study received EBRT and/or brachytherapy. EBRT was performed with either a 2-field technique, a 4-field technique, or with intensity modulated therapy (IMRT) depending on the treatment era. Brachytherapy was performed with either low dose rate (LDR) or high dose rate (HDR) methods. A total of 7 patients $(11.1 \%)$ received EBRT alone, while 30 patients (47.6\%) and 18 patients (28.6\%) received EBRT with HDR brachytherapy and EBRT with LDR brachytherapy, respectively. Seven patients (11.1\%) received HDR alone, while 1 patient $(1.6 \%)$ received LDR alone. Fifteen patients $(23.8 \%)$ had surgery as a component of their treatment and 14 patients $(22.2 \%)$ received concurrent chemotherapy. The most commonly prescribed chemotherapy regimen was weekly cisplatin at a dose of $40 \mathrm{mg} / \mathrm{m}^{2}$.

\section{Data collection}

Patient data was gathered by means of a retrospective chart review, after obtaining IRB (Institutional Review Board) approval. Acute and late toxicities were scored according to the Common Terminology Criteria for Adverse Events version 3 (CTCAE v3.0). Radiobiologic comparison of different treatment methods was performed by calculating the biologic effective dose, $\mathrm{BED}_{3}$ and $\mathrm{BED}_{10}$, using the following formula:

$$
\mathrm{BED}=\mathrm{nd}[1+\mathrm{d} /(\alpha / \beta)]
$$

where $\alpha / \beta=10$ for acute effects and $\alpha / \beta=3$ for late effects.

Statistical analyses were performed using IBM SPSS Statistics Version 19.0.0 (IBM Corporation, Armonk, NY, USA). Actuarial LC, DFS, OS, and late toxicity rates were calcu- lated using the Kaplan-Meier method. A logrank test was performed to detect significant differences in survival related to patient and tumor characteristics, as well as treatment techniques. Correlation analyses were performed using the Pearson correlation test.

\section{Results \\ Patient population}

The study population included 57 patients with squamous cell carcinoma and 6 patients with adenocarcinoma (Table 2). The median age at diagnosis was 70 years (range 33-84 years). The median follow up time was 44.2 months. In total, there were 48 patients $(76.2 \%)$ with stage I-II disease and 15 patients $(23.8 \%)$ with stage III-IVA disease (Table 1). The extent of disease at diagnosis was estimated using tumor size $(\mathrm{cm})$ and length of vaginal involvement $(1 / 3,2 / 3$, or whole vagina). Thirty-seven patients had a tumor size $<4 \mathrm{~cm}$, while 24 patients had a tumor size $\geq 4 \mathrm{~cm}$. Thirty-eight patients had approximately $1 / 3$ of the length of the vagina involved, while 14 and 9 patients had $2 / 3$ and whole vaginal involvement, respectively. Tumor location was approximately equal when divided into the following sub-sites: apex, upper $1 / 3$, middle $1 / 3$, and lower $1 / 3$, with 45 patients having a tumor which occupied multiple subsites (Table 2).

\section{Outcomes based on tumor and patient characteristics}

Comparison of patients with early stage vaginal cancer (stages I and II) versus those with locally advanced disease (stages III and IVA) demonstrated a superior 5-year OS (73.3 vs. $34.4 \%, p=0.032$ ) (Fig. 1). There was no statistically significant difference noted in 5-year DFS or 5-year LC between the two stage groupings. In addition to FIGO stage, we analyzed the impact of primary tumor size $(<4 \mathrm{~cm}$ vs. $\geq 4 \mathrm{~cm}$ ) and length of vaginal involvement $(<1 / 3 \mathrm{vs} . \geq 1 / 3)$ on disease control and survival. The cutoff size of $<4 \mathrm{~cm}$ was selected based on previously published literature [4]. In our patient population, there were trends toward improved outcomes for patients with tumor size $<4 \mathrm{~cm}$, which did not reach statistical significance. This included 5 -year OS $(p=0.098), 5$-year DFS $(p=0.116)$ and 5-year LC $(p=0.078)$ (Table 2$)$. When comparing patients with tumor involving less than $1 / 3$ of the vaginal canal to those with more extensive disease, significantly better outcomes were noted for 5-year LC (86.9 vs. $60.4 \%, p=0.018$ ) and 5-year DFS (84.0 vs. $52.4 \%, p=0.007$ ) (Fig. 2). There was also a trend toward improved 5-year OS between the two groups (75.6 vs. $48.7 \%, p=0.081$ ). Tumor location within the vagina also 
Table 2. Outcomes based on patient, tumor and treatment characteristics

\begin{tabular}{|c|c|c|c|c|c|c|c|}
\hline Characteristic & $n$ & 5-yr LC (\%) & $p$-value & 5-yr DFS (\%) & $p$-value & $5-y r$ OS (\%) & $p$-value \\
\hline \multicolumn{8}{|l|}{ Stage } \\
\hline Early stage $(I+I I)$ & 48 & 80.3 & \multirow{2}{*}{0.102} & 73.9 & \multirow{2}{*}{0.252} & 73.3 & \multirow{2}{*}{0.032} \\
\hline Locally advanced (III + IV) & 15 & 61.1 & & 58.7 & & 34.4 & \\
\hline \multicolumn{8}{|l|}{ Tumor size } \\
\hline$<4 \mathrm{~cm}$ & 37 & 84.7 & \multirow{2}{*}{0.078} & 79.6 & \multirow{2}{*}{0.116} & 75.6 & \multirow{2}{*}{0.098} \\
\hline$\geq 4 \mathrm{~cm}$ & 24 & 61.5 & & 56.8 & & 50.9 & \\
\hline \multicolumn{8}{|l|}{ Tumor location } \\
\hline Apex & 37 & 76.6 & 0.716 & 73.6 & 0.419 & 67.6 & 0.654 \\
\hline Upper $1 / 3$ & 41 & 75.4 & 0.778 & 72.8 & 0.375 & 67.4 & 0.388 \\
\hline Middle $1 / 3$ & 33 & 64.3 & 0.023 & 55.8 & 0.004 & 49.2 & 0.050 \\
\hline Lower $1 / 3$ & 26 & 72.5 & 0.752 & 66.7 & 0.824 & 61.9 & 0.354 \\
\hline \multicolumn{8}{|l|}{ Degree of involvement } \\
\hline Whole vaginal & 9 & 77.8 & 0.603 & 77.8 & 0.394 & 68.2 & 0.856 \\
\hline $2 / 3$ & 14 & 46.9 & 0.000 & 34.3 & 0.000 & 25.6 & 0.015 \\
\hline $1 / 3$ & 38 & 86.9 & 0.018 & 84.0 & 0.007 & 75.6 & 0.081 \\
\hline \multicolumn{8}{|l|}{ Brachytherapy technique } \\
\hline HDR & 36 & 81.8 & \multirow{2}{*}{0.585} & 72.3 & \multirow{2}{*}{0.196} & 61.0 & \multirow{2}{*}{0.088} \\
\hline LDR & 19 & 89.2 & & 88.8 & & 82.9 & \\
\hline \multicolumn{8}{|l|}{ Age } \\
\hline$<65$ & 26 & 80.2 & \multirow{2}{*}{0.441} & 76.0 & \multirow{2}{*}{0.391} & 65.2 & \multirow{2}{*}{0.250} \\
\hline$\geq 65$ & 37 & 72.8 & & 66.9 & & 63.9 & \\
\hline \multicolumn{8}{|l|}{ Histology } \\
\hline Squamous & 57 & 77.2 & \multirow{2}{*}{0.250} & 69.7 & \multirow{2}{*}{0.725} & 63.6 & \multirow{2}{*}{0.982} \\
\hline Adenocarcinoma & 6 & 100.0 & & 83.3 & & 75.0 & \\
\hline \multicolumn{8}{|l|}{ Chemotherapy } \\
\hline Yes & 14 & 76.9 & \multirow{2}{*}{0.555} & 76.9 & \multirow{2}{*}{0.857} & 37.5 & \multirow{2}{*}{0.335} \\
\hline No & 49 & 77.6 & & 71.2 & & 67.0 & \\
\hline
\end{tabular}

$L C$ - local control, DFS - disease free survival, OS - overall survival, HDR - high dose rate, $L D R$ - low dose rate

impacted outcomes in this series of patients. Tumors located in the apex, upper $1 / 3$, or lower $1 / 3$ of the vagina did not demonstrate any differences in 5-year OS, DFS, or LC (Table 2). However, patients with tumors occupying the

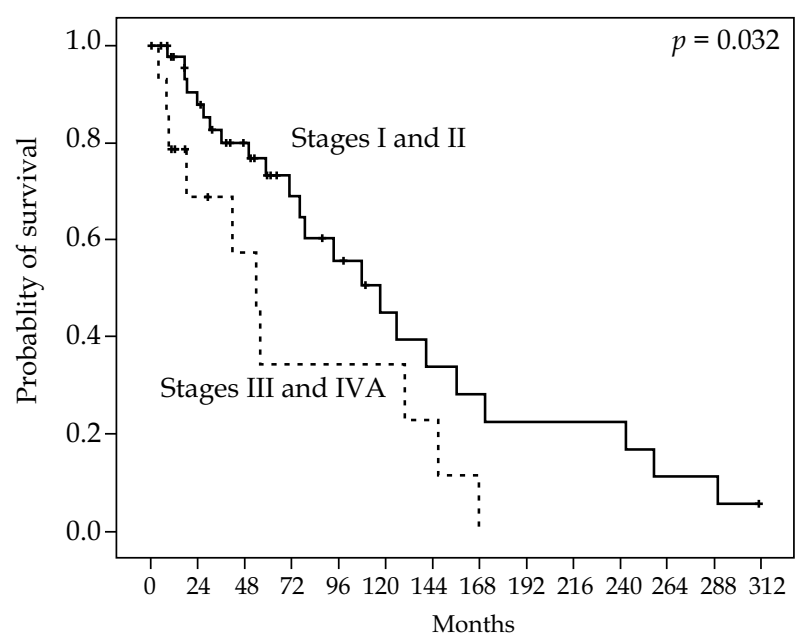

Fig. 1. Kaplan-Meier overall survival curve comparing patients with early stage disease (stages I and II) versus patients with locally advanced disease (stages III and IVA) $(p=0.032)$ middle $1 / 3$ of the vagina had significantly worse 5 -year OS $(p=0.050)$, DFS $(p=0.004)$, and LC $(p=0.023)$ when compared to tumors occupying the apex, upper $1 / 3$, or lower $1 / 3$ of the vagina (Fig. 3). Comparing those patients with an age of less than 65 years to those with an age greater than 65 years, there were no differences found in 5-year LC, DFS, or OS. Additionally, no differences were noted in outcomes when comparing squamous cell carcinoma vs. adenocarcinoma (Table 2).

\section{Outcomes based on treatment techniques}

This series contained data on patients spanning over 26 years, and thus patients were treated via a variety of methods depending on their era of treatment. Those treated with LDR brachytherapy, compared with HDR brachytherapy, had a trend toward improved OS at 5 years, but this difference was not significant ( 82.9 vs. $61.0 \%, p=0.088)$. When analyzing the outcomes for 5-year DFS $(p=0.196)$ and LC $(p=0.585)$, this trend was no longer evident. The impact of total prescribed radiation dose on outcome also was analyzed using a $\mathrm{BED}_{10}$ calculation to standardize the dose comparison. The median $\mathrm{BED}_{10}$ in this population was 86.1 Gy (range 36.0-146.6 Gy). A Pearson correlation test demonstrated a positive, nearly significant correlation of increasing $\mathrm{BED}_{10}$ with 5-year LC $(r=0.223, p=0.086)$, and a positive, but not significant correlation with 5-year DFS 

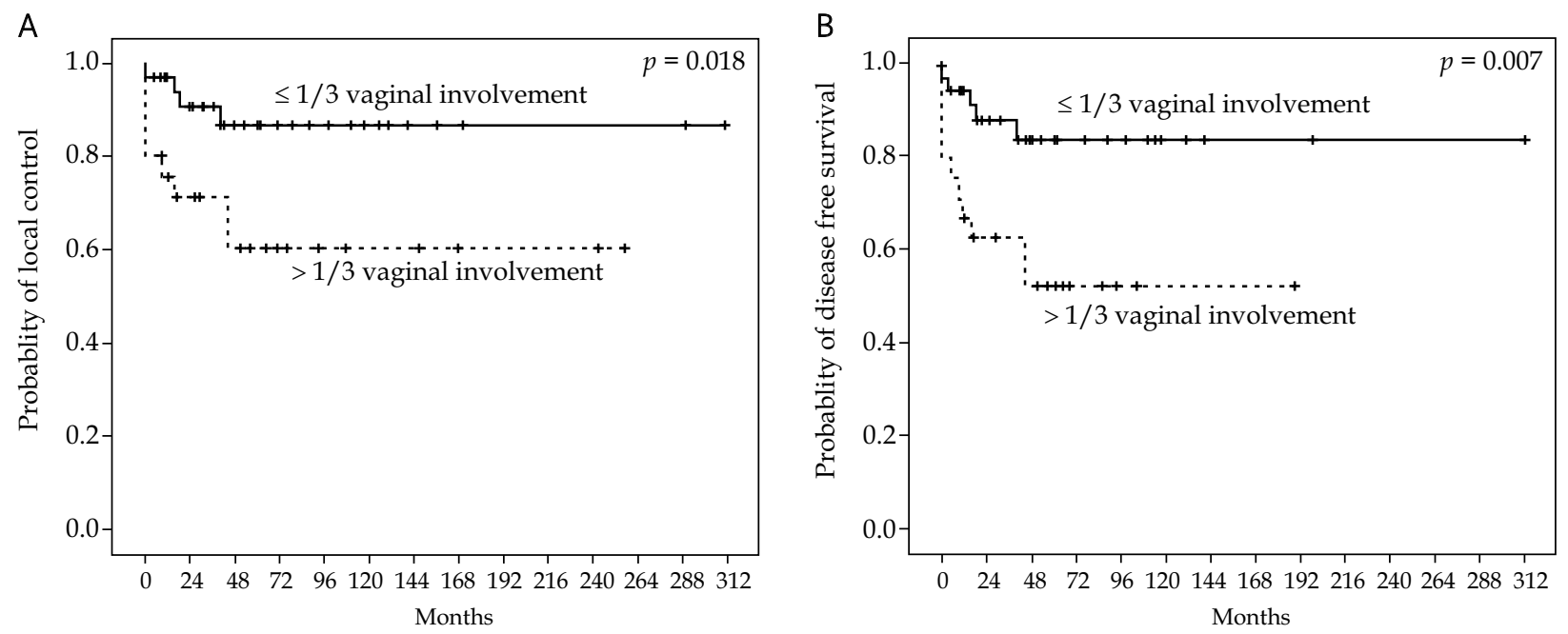

Fig. 2. Kaplan-Meier curves demonstrating (A) improved local control $(p=0.018)$ and $\mathbf{( B )}$ disease free survival $(p=0.007)$ in patients with $1 / 3$ or less vaginal canal involvement by tumor versus patients with greater the $1 / 3$ vaginal involvement
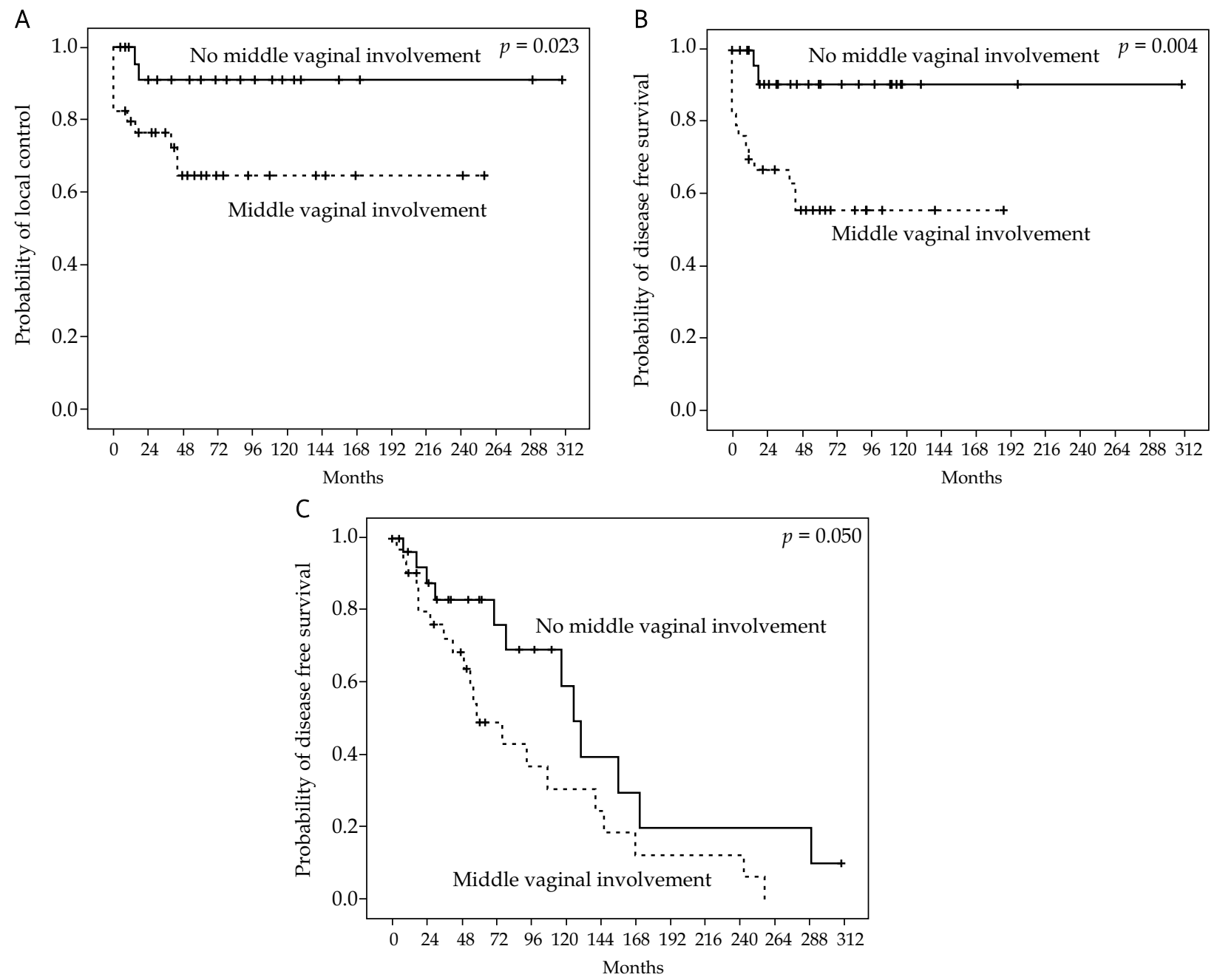

Fig. 3. Kaplan-Meier curves for (A) local control $(p=0.023)$, (B) disease free survival $(p=0.004)$, and (C) overall survival $(p=0.050)$, comparing patients with middle $1 / 3$ vaginal involvement versus those patients without $1 / 3$ middle vaginal involvement 


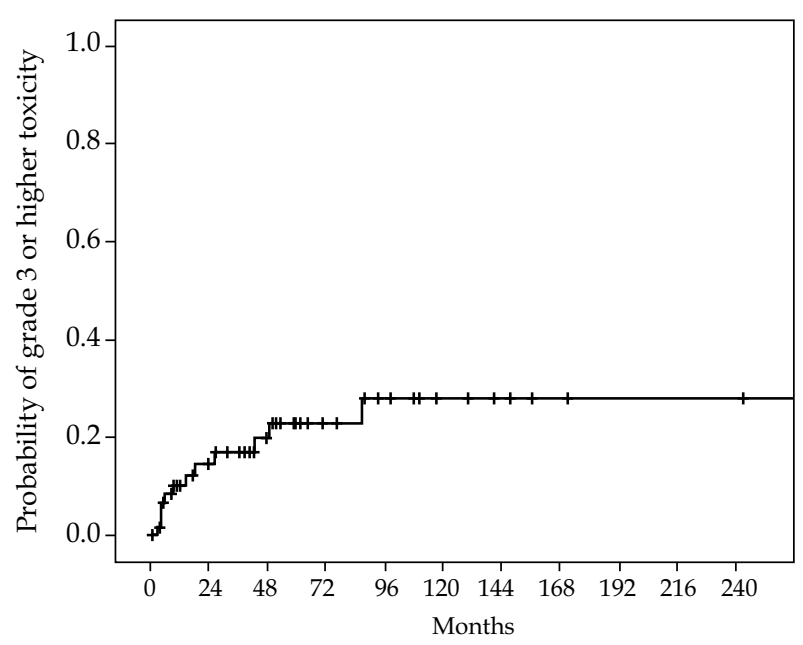

Fig. 4. Kaplan-Meier curve demonstrating the actuarial rate of grade 3 or higher late toxicities

$(r=0.177, p=0.176)$. Of the 14 patients who received concurrent chemotherapy, 6 patients had early stage disease while 8 patients had locally advanced disease. There was no difference in 5-year OS for those not receiving concurrent chemotherapy versus those receiving chemotherapy $(p=0.335)$. Additionally, there was no difference in 5-year DFS $(p=0.857)$ or LC $(p=0.555)$.

\section{Toxicities}

There where 10 patients (15.9\%) who manifested acute grade 3 or higher toxicities, most commonly radiation dermatitis or vaginal mucositis $(n=8)$. Thirteen patients $(20.6 \%)$ developed grade 3 or higher late toxicities, most commonly vaginal stenosis and/or necrosis $(n=6)$. Other late toxicities included rectal bleeding (4), skin/soft tissue necrosis (2), rectovaginal fistula formation (1), vesicovaginal fistula formation (1), pelvic insufficiency fracture (1), hydronephrosis (1), and hemorrhagic cystitis (1). There was one late grade 5 toxicity, which was a fatal hemorrhage from a rectovaginal fistula 30 months after completing therapy. This patient had pelvic radiation therapy 8 years prior to her diagnosis of vaginal cancer, and also had a total of 3 other grade 3 toxicities develop prior to the hemorrhage. The 5-year actuarial rate of grade 3-5 late toxicities in this study was $23.1 \%$ (95\% CI: 10.6-35.6\%) (Fig. 4). There was a nearly significant correlation with highest grade of late toxicity and $\mathrm{BED}_{3}(r=0.241, p=0.059)$, based on a Pearson correlation analysis.

Administration of concurrent chemotherapy did not have an impact on acute or late toxicities. A Pearson correlation test demonstrated no association regarding the use of chemotherapy and highest grade of late toxicity $(r=0.004, p=0.974)$ or number of grade 3-5 late toxicities $(r=0.107, p=0.414)$.

\section{Discussion}

Vaginal cancer is a rare malignancy for which prospective randomized clinical studies are lacking. Only a small number of retrospective reviews are available to outline risk factors for aggressive disease and offer guidance in mana- gement of these patients [1,2,4,5,7-10]. In our series, we clearly demonstrate superior OS in patients with stages I-II disease, compared to patients with locally advanced disease. This is consistent with several other retrospective series, which also have demonstrated inferior OS and disease control in patients with advanced disease at diagnosis $[1,4$, $5,8,11]$.

Increasing tumor size $(>4 \mathrm{~cm})$ has been shown to predict for inferior disease control and disease-specific survival at 5 years [4]. In our patient population, we found statistically insignificant trends toward inferior LC, DFS and OS at 5 years in patients with tumor size $\geq 4 \mathrm{~cm}$. Additionally, when analyzing by extent of vaginal involvement, we found superior outcomes in those patients whose primary tumors involved $\leq 1 / 3$ of the vaginal canal. This supports the previously published literature depicting better outcomes in those patients with smaller tumors [4].

Furthermore, tumor location within the vagina has been hypothesized to have prognostic implications. Frank et al. [4] and Chyle et al. [9] suggested a trend toward inferior outcomes with middle/distal vaginal tumors compared to proximal and apical vaginal tumors. However, our data demonstrated markedly inferior outcomes for patients with tumors located in the middle $1 / 3$ of the vagina. This may be explained by the extent of vaginal length involved by the primary tumor, as 25 of the 32 patients $(78.1 \%)$ in our series with middle $1 / 3$ tumors had multiple levels of vaginal involvement. From the aforementioned analysis comparing $\leq 1 / 3$ vaginal involvement versus $>1 / 3$ involvement, there was a clear benefit to having lesser vaginal involvement, and thus this observation aids in explaining the significantly inferior outcomes in those patients with tumors occupying the middle $1 / 3$ of the vagina. Additionally, our study did not replicate some previously published data suggesting inferior outcomes for distal vaginal involvement. It is important to note that not all series have suggested a relationship between tumor location and prognosis [1,12].

Treatment techniques employed in our patient population were variable due to the 26-year period encompassed by our study, and also because treatment of vaginal cancer is highly individualized based on the location and extent of disease. We did not find a significant association between brachytherapy method (LDR vs. HDR) and outcome. Our study did suggest a potential, but not statistically significant trend, for greater $\mathrm{BED}_{10}$ values correlating with improved local control and disease-free survival. To our knowledge, our series is the first to examine the impact of BED upon outcome in vaginal cancer. Frank et al. [4] have previously shown that a prescription dose of $\geq 75$ Gy (EBRT + LDR brachytherapy) does not correlate with improved pelvic disease control or disease-specific survival. Perez et al. [10], however, found that patients with stage IIA disease (based on FIGO staging with minor modification dividing stage II into IIA with paravaginal extension or IIB with parametrial involvement) had improved LC when the tumor received doses greater than 55 Gy. Furthermore, patients with stage IIB and III disease had improved tumor control with medial parametrial dose greater then $65 \mathrm{~Gy}$ and lateral parametrial dose (encompassing the pelvic nodes) greater than $50 \mathrm{~Gy}$. 
Presently, the role of chemotherapy in the treatment of vaginal cancer is poorly defined and based primarily on extrapolation from cervical cancer series [13-15]. Samant et al. [2] retrospectively analyzed the feasibility of concurrent cisplatin based chemoradiation in 12 patients, and found that $92 \%$ of patients completed all cycles of chemotherapy at full dose, with no hospitalizations in the acute period. They report satisfactory outcomes with a 5-year OS of $66 \%$ and 5 -year local-regional progression-free survival of $92 \%$. Our study did not shown any benefit with the addition of chemotherapy. In fact, there was an insignificant trend toward inferior OS in the 14 patients receiving chemotherapy in this study (Table 2). Similarly, Sinha et al. [1] demonstrated a trend toward inferior DFS with the addition of chemotherapy ( 55 vs. $86 \%, p=0.051$ ). Frank et al. [4] concluded that the efficacy of concurrent chemoradiotherapy could not be determined from their series, but suggested that patients with high risk disease (stage III-IVA) and a good performance status may benefit from the concurrent treatment regimen. Factors such as small sample size and physician bias toward prescribing chemotherapy for more advanced tumors may obscure the benefits of concurrent chemotherapy in these retrospective analyses. To date, little data has been published regarding severe toxicities after treatment of vaginal cancer. Our study demonstrated a 5-year actuarial grade 3-5 toxicity rate of $23.1 \%$ (95\% CI: 10.6-35.6). The high actuarial toxicity rate can, in part, be attributed to the previously mentioned patient who expired from a grade 5 hemorrhage, also having incurred 3 other grade 3 toxicities. This was due to the fact that she had previously received pelvic radiation therapy in the past for an unrelated malignancy.

Concurrent chemotherapy did not impact toxicity in our patient population. Frank et al. [4] showed that the incidence of major complications increases with stage of disease, likely a function of more aggressive treatment approaches. Recently, de Crevoisier et al. found that bladder toxicity correlates with anterior tumor location, vaginal toxicity correlates with FIGO stage and length of vaginal involvement, and grade 2-3 urinary or digestive toxicity correlates with the total reference air kerma of the brachytherapy plan [5]. Our data demonstrates a near significant correlation with late toxicity and $\mathrm{BED}_{3}$. This suggests the presence of a potential therapeutic window, given the previously discussed trend towards a positive correlation between superior tumor control with a greater $\mathrm{BED}_{10}$. Future analysis on the impact of $\mathrm{BED}_{3}$ and $\mathrm{BED}_{10}$ upon late toxicity and tumor control, using a larger patient population, may be beneficial in this regard. Additionally, MRI and CT-guided brachytherapy planning is anticipated to aid in optimizing tumor coverage while minimizing dose to adjacent critical structures, thereby reducing toxicity.

Presently, our institutional approach includes multidisciplinary evaluation and individualization of therapy based upon prognostic features, such as FIGO stage and the size/extent of the primary tumor, as well as tumor location. Brachytherapy alone is reserved for those patients with in situ disease or very early stage vaginal cancer, as supported by Seeger et al. [16]. The majority of patients, are treated with 45 Gy EBRT to the pelvis \pm inguinal lymph nodes, followed by a brachytherapy boost to the primary tumor and an EBRT boost to any grossly involved regional lymph nodes. The clinical decision regarding method of brachytherapy (LDR or HDR) depends on tumor size, location, depth of invasion, response to EBRT, and need for interstitial implant versus an intracavitary procedure. Taking into account the above data, we utilize concurrent weekly cisplatin at $40 \mathrm{mg} / \mathrm{m}^{2}$ in healthy patients with locally aggressive tumors.

The authors recognize the limitations of this study including small sample size, retrospective nature of the analysis, and extended treatment era resulting in patients treated with multiple different techniques. Unfortunately, given the rarity of primary vaginal cancer, these limitations are not unique to our series.

\section{Conclusions}

Overall, our data confirms that increasing FIGO stage and primary tumor extent predict for inferior outcomes, indicating the need for aggressive multidisciplinary management. A definitive radiation approach is preferable, combining the use of EBRT and LDR or HDR brachytherapy, depending on the clinical scenario. The benefit of concurrent chemotherapy is of debate. Based on extrapolation from other gynecologic malignancies, we continue to prescribe weekly cisplatin for patients with advanced tumors and an adequate performance status. In the future, improved outcomes may be achieved by more rigorously defining the $\mathrm{BED}_{10}$ for tumor control and by utilizing IMRT and image-guided brachytherapy to optimize dose distribution.

\section{Conflict of interest notification}

The authors have no conflicts of interest to disclose in direct relation to this publication. Margaret Straub, PA-C has institutional grant funding for a randomized controlled trial for evaluation of a multifaceted vaginal renewal program utilizing vibrating wands in gynecologic cancer patient experiencing sexual dysfunction. Kristin Bradley, MD is the author of two Up-To-Date sections, has received funding from the National Childhood Cancer Foundation for study CA098543-06, and is also the study chair for ACNS0222, a phase II study of Motexafin-Gadolinium and involved field radiation therapy for intrinsic pontine glioma of childhood.

Material was partially presented at the 2011 American Brachytherapy Society Annual Meeting in San Diego, California, USA.

\section{References}

1. Sinha B, Stehman F, Schilder J et al. Indiana university experience in the management of vaginal cancer. Int J Gynecol Cancer 2009; 19: 686-693.

2. Samant R, Lau B, Choan E et al. Primary vaginal cancer treated with concurrent chemoradiation using cis-platinum. Int J Radiat Oncol Biol Phys 2007; 69: 746-750.

3. American Cancer Society. Cancer facts \& figures 2010. American Cancer Society, Atlanta 2010.

4. Frank SJ, Jhingran A, Levenback C et al. Definitive radiation therapy for squamous cell carcinoma of the vagina. Int J Radiat Oncol Biol Phys 2005; 62: 138-147. 
5. De Crevoisier R, Sanfilippo N, Gerbaulet A et al. Exclusive radiotherapy for primary squamous cell carcinoma of the vagina. Radiother Oncol 2007; 85: 362-370.

6. Kushner DM, Fleming PA, Kennedy AW et al. High dose rate (192)Ir afterloading brachytherapy for cancer of the vagina. Br J Radiol 2003; 76: 719-725.

7. Beriwal S, Heron DE, Mogus R et al. High-dose rate brachytherapy (hdrb) for primary or recurrent cancer in the vagina. Radiat Oncol 2008; 3: 7; doi: 10.1186/1748-717X-3-7.

8. Hegemann S, Schafer U, Lelle R et al. Long-term results of radiotherapy in primary carcinoma of the vagina. Strahlenther Onkol 2009; 185: 184-189.

9. Chyle V, Zagars GK, Wheeler JA et al. Definitive radiotherapy for carcinoma of the vagina: outcome and prognostic factors. Int J Radiat Oncol Biol Phys 1996; 35: 891-905.

10. Perez CA, Grigsby PW, Garipagaoglu M et al. Factors affecting long-term outcome of irradiation in carcinoma of the vagina. Int J Radiat Oncol Biol Phys 1999; 44: 37-45.

11. Tewari KS, Cappuccini F, Puthawala AA et al. Primary invasive carcinoma of the vagina: Treatment with interstitial brachytherapy. Cancer 2001; 91: 758-770.

12. Perez CA, Camel HM, Galakatos AE et al. Definitive irradiation in carcinoma of the vagina: Long-term evaluation of results. Int J Radiat Oncol Biol Phys 1988; 15: 1283-1290.

13. Morris M, Eifel PJ, Lu J et al. Pelvic radiation with concurrent chemotherapy compared with pelvic and para-aortic radiation for high-risk cervical cancer. N Engl J Med 1999; 340: 11371143.

14. Keys HM, Bundy BN, Stehman FB et al. Cisplatin, radiation and adjuvant hysterectomy compared with radiation and adjuvant hysterectomy for bulky stage ib cervical carcinoma. N Engl J Med 1999; 340: 1154-1161.

15. Rose PG, Bundy BN, Watkins EB et al. Concurrent cisplatinbased radiotherapy and chemotherapy for locally advanced cervical cancer. N Engl J Med 1999; 340: 1144-1153.

16. Seeger AR, Windschall A, Lotter $\mathrm{M}$ et al. The role of interstitial brachytherapy in the treatment of vaginal and vulvar malignancies. Strahlenther Onkol 2006; 182: 142-148. 